\title{
Some Analyses of Slow Potentials of Toad's Retina
}

\author{
By \\ Eizo Yamashita \\ (山下 栄三) \\ From the Physiological Laboratory of Prof. K. Motokawa \\ Tohoku University, Sendai
}

(Received for publication, April 15, 1959)

Since the discovery of the electroretinogram by Holmgren in 1865 workers have attempted to clarify the mechanism of its generation. The ERG is a compound response. Granit ${ }^{1)}$ separated three processes PI, PII and PIII by the effects of narcotics and other chemical agents, and they have been admitted widely to-day. The sources of these components within the retina, however, were not clear, though Granit $^{11}$ and others reported some opinions about them. Recent development of the microelectrode technique has made possible the recording of focal responses in every depth of the retina with microillumination. Tomita ${ }^{2)}$, Svaetichin ${ }^{3)}$, Brindley ${ }^{4}$ and other workers have analyzed retinal responses by this method. The inverted retina preparation devised by Svaetichin is convenient to use for this purpose. Noell ${ }^{51}$, on the other hand, has analyzed the ERG from the electrophysiological and histological effects of azides and iodates. Svaetichin ${ }^{2)}$ and Brindley) claimed that the ERG was elicited from the receptor layer and that focal responses made no contribution to the usual ERG. Svaetichin ${ }^{6)}$ ascribed components of opposite polarity to cones and rods. Noell ${ }^{5}$ regarded the receptor layer as the sources of PII and PIII, while Tomita ${ }^{788}$ claimed that at least a part of the ERG originated in secondary neurons, especially bipolar cells.

In the present investigation some experiments on slow potentials of toad's retina were done with the microtechnique, and some analysis was attempted of the so obtained experimental results.

\section{EXPERIMENTAL}

\section{Method}

Experiments were made on retinae of toads (Bufo vulgaris). Preparations were made in daylight or under rather weak room illumination. Toads were kept in a dark room several hours before ophthalmectomy. After removing the pigment epithelium an inverted retina was mounted 
with the receptor side upwards on a piece of black cloth soaked with saline. An Ag-AgCl electrode was set beneath the cloth to be used as an indifferent electrode. A KCl-filled microelectrode was inserted from the surface of the receptor side, but in some experiments (see Fig. 1) a wick electrode was used. The intensity of light was about 4,000 lux and in several experiments microillumination was made with a small light spot $100 \times 150 \mu$ in size. When a smaller spot was used in a part of experiments, an image of the filament of a light source placed on the ocular side of a microscope was focused on the retina through the microscope. Thus a spot as small as $20 \mu$ in diameter and 6,000 lux in intensity was obtained. The recording was made by means of a D.C.-amplifier with a cathode-follower input and a cathoderay oscilloscope. Sometimes a C.R.-coupled amplifier with a time-constant of 1.5 seconds and a penwriter were used.

\section{Results}

\section{Contribution of the pigment epithelium to slow potentials}

As the pigment epithelium is usually removed in the inverted retina preparation, the question arises as to whether the response obtained from the inverted retina is unnatural. So the typical slow potential of the inverted retina was compared with that obtained from the eyebulb preparation in which the pigment epithelium remained intact. Fig. 1, A shows a response to even illumination. In this experiment a wick-electrode was placed on the inner surface of the retina, and the positivity of the electrode was directed upwards. An inverted retina preparation was prepared from the same eye after taking record $\mathrm{A}$, and the record shown in Fig. 1, a was obtained. For convenience of comparison the negativity of the wickelectrode on the receptor side is directed upwards in this record. In general, both records $\mathrm{A}$ and $a$ resemble one another. The upward deflection at " on" is the b-wave, which can be preceded by a downward deflection usually called the a-wave. In the usual ERG the b-wave is followed by a slow c-wave. It is to be noted that the record which was obtained from the inverted retina without the pigment epithelium lacks the c-wave. The pair of records $\mathrm{B}$ and $b$ represent another example. The record $\mathrm{B}$ obtained from the eyebulb shows a remarkable $\mathrm{c}$-wave, while it is absent in the record $b$. The difference was investigated on more than ten preparations and there were little departures.

Noell ${ }^{5}$ claimed that c-wave was due to the pigment epithelium, and the results obtained above support his opinion.

The amplitude of response, especially of the off-response (d-wave) was often larger in the inverted retina. Some inverted retinae showed a deep a-wave and a small b-wave, but this seemed to be caused from some 

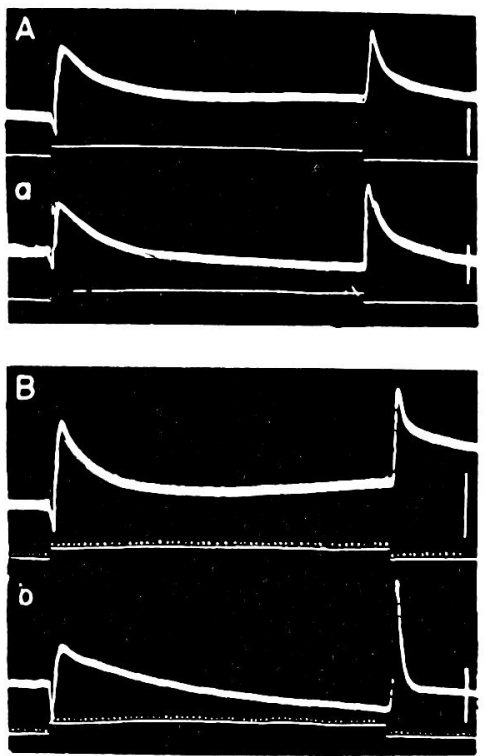

Fig. 1. A and B show responses recorded from opened excised eye. Positivity of wick-electrode placed on inner surface of retina is directed upwards. $a$ and $b$ show responses recorded from inverted retina. Negativity of wick-electrode placed on outer surface of retina is directed upwards. Vertical bar indicates $300 \mu \mathrm{V}$, and stimulus duration is about 6 seconds.

damage in removing the pigment epithelium.

2. Types of response

In the following experiments exclusively inverted retinae were used, and the polarity of response was referred to the microelectrode placed on the receptor side of the preparation. As has been shown in Fig. 1, the a- and b-waves of ERG correspond with positivity and negativity of the microelectrode on the receptor side respectively, but such notations $a, b$, $\mathrm{c}$ and $\mathrm{d}$ will not be used for records obtained from inverted retinae, because the properties of such records are different in many respects from those of the usual ERG, for example, the ERG is diffuse in character, while the response recorded by a microelectrode to microillumination is focal in character. The positivity is directed upwards in all the following records.

Under some conditions responses lacking the negative component were obtained (see Fig. 2). When the pigment epithelium was removed carelessly so that the surface of the receptor side was possibly damaged, this type of response was obtained to even illumination. This type will be called positive type. Sometimes a part of retina showed this type and the other parts showed normal or negative type. In winter and especially in early 
spring inverted retinae of toads which passed hibernation were so apt to show the positive type that it was difficult to obtain the normal type in spite of careful treatment. The possibility of obtaining the normal type was not increased even if toads were kept at room temperature of $20^{\circ} \mathrm{C}$ about ten hours before experiments, or preparations were warmed to $25^{\circ} \mathrm{C}$. Svaetichin $^{3)}$ reported that the b-wave of the frog's retina was not absent under such low temperature as $1^{\circ} \mathrm{C}$. Several toads were fed on toad's liver or injected 0.5 c.c. of liver oil into the peritoneal cavity 48 hours before experiment, but none of their retinae showed a complete negative type. To pour saline on a preparation showing responses of the positive type was found sometimes useful to change its response type. Bernhard9) and Deane et al. ${ }^{10)}$ reported a similar type of ERG of turtles which were in hibernation. But responses recorded from opened excised eyes of toads did not show the positive type so often as responses of inverted retinae. A piece of retina minced about $1 \mathrm{~mm}^{2}$ in area showed responses of the positive type as investigated by Ottoson and Svaetichin ${ }^{11}$.

In some preparations showing responses of the positive type a negative deflection appeared at cessation of light as in Fig. 2, B. When the duration of the stimulus was short, no "off" "response appeared in normal type but did in positive type.

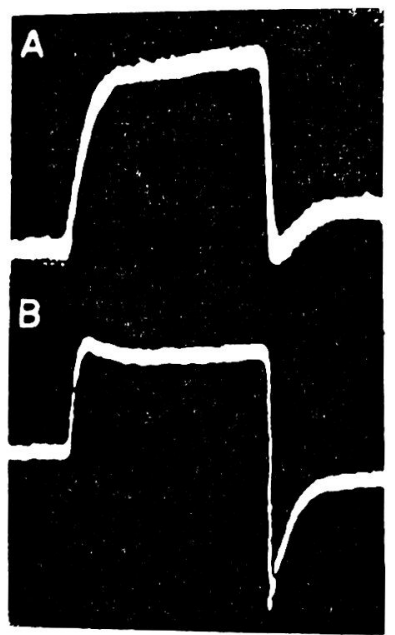

Fig. 2. Some response types of inverted retina. Positivity of electrode placed on outer surface of retina is directed upward.

Next, responses to microillumination will be mentioned. As has been shown in Fig. 1, the response recorded from the surface of the inverted retina to even illumination was very similar to the usual ERG and this relation changed little when a spot of stimulus light as small as $20 \mu$ in 
diameter was used (see Fig. 4 or Fig. 7). The type of response to microillumination usually suffered from a considerable change by touching the surface of the inverted retina with a piece of dry filter paper; Responses of the negative type before the treatment turned into responses of the positive type. Tomita found a similar effect (personal communication), and Pilz et al. ${ }^{12)}$ reported also a similar change which they considered to be due to damage. From what has been observed above it is likely that the positive type appears when the receptor surface is damaged in some way or other.

3. Type of response and adaptation state

Sometimes a negative going deflection was superimposed on the basic positive response (see Fig. 3A). In this experiment the stimulus light

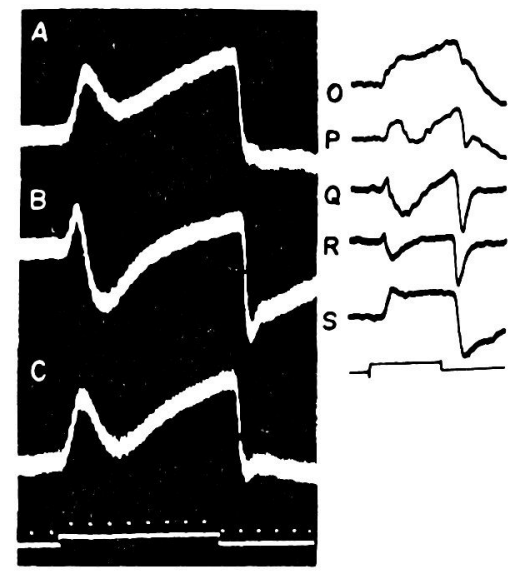

Fig. 3. Effect of light adaptation on responses of inverted retina.

A, C : Responses under dark adaptation.

B : Response under background illumination of 20 lux.

O : Dark; P : 2 lux; Q: 5 lux; R: 20 lux; S : 5 lux. Positivity upwards. Time mark: $10 \mathrm{cps}$.

spot was $1 \times 1 \mathrm{~mm}$. in size. The negative response became larger when the preparation was light-adapted to background illumination of 20 lux (see Fig. 3B). When the adapting light was turned off, a response of the original shape was obtained again (see Fig. 3G). Such a difference due to background illumination was distinct in preparations made from toads which had been light-adapted for several hours. In spring one preparation made from a toad kept long in sunshine showed response of the positive type in dark adaptation, but those of negative type in light adaptation. The records in Fig. 3, O-S show this relation. The stimulus light spot was $20 \mu$ in diameter and 6,000 lux in intensity in all the records and the intensity of background illumination was $0,2,5$ and 20 lux for $\mathrm{O}, \mathrm{P}, \mathrm{Q}$ 
and $\mathrm{R}$ respectively, and it was reduced to 5 lux in $\mathrm{S}$ after $\mathrm{R}$ had been recorded. But in $\mathrm{S}$ the negative deflection was only slight compared with $Q$, although background illumination for $Q$ and $S$ was identical.

4. Properties of focal responses

It seemed that the type of response was characteristic of the locus from which the potential was recorded. A microelectrode placed on a locus damaged by a touch with filter paper picked up responses of positive type, while a microelectrode placed on an intact part around the damaged part showed normal negative responses. The difference in type of the responses at the damaged and intact parts did not disappear when the preparation was subjected to even illumination. This finding suggests that a microelectrode can pick up focal responses. The question arose to what extent a focal response at a locus spread into the surrounding area. To answer this question, the following experiments were carried out. A microillumination of $20 \mu$ in diameter was used. The intensity was 6,000 lux, and the background illumination 15 lux. First a locus on which the microelectrode was placed was illuminated and a normal response consisting of remarkable negative "on" and "off" deflections was obtained (see record labeled $0 \mathrm{~mm}$. in Fig. 4). When the microillumination was dis-

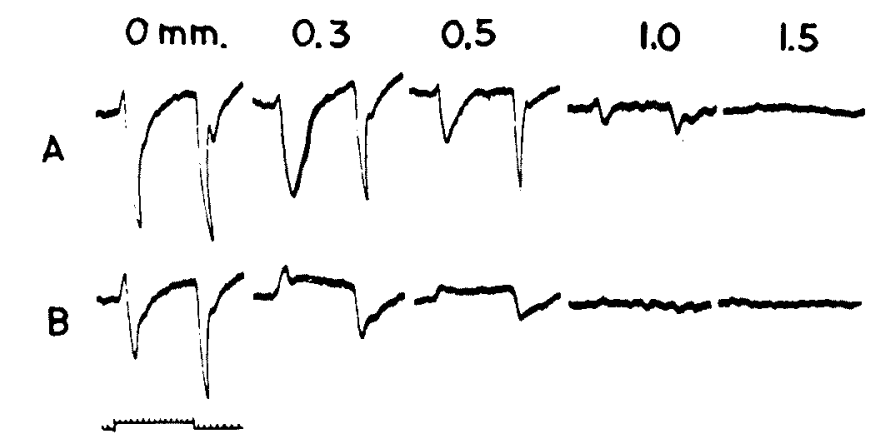

Fig. 4. A: Dependence of response on distance between recording electrode and microillumination. Numerals above records show distances. B : Similar dependence when mechanical cut was made at $0.3 \mathrm{~mm}$. Time scale indicates $100 \mathrm{msec}$.

placed to points $0.3,0.5,1.0$ and $1.5 \mathrm{~mm}$. distant from point 0 , the records labeled $0.3,0.5,1.0$ and 1.5 in series A of Fig. 4 were obtained respectively. It is apparent from these records that the magnitude of response decreases with increasing distance from the illuminated locus, and that no response is perceived at a distance of $1.5 \mathrm{~mm}$.

Next, a shallow cut was made with a blade of razor at a point $0.3 \mathrm{~mm}$. from the electrode. When illumination was again centered on the electrode, a response very similar to that taken before was obtained, although 
the amplitude was a little smaller (see series B of Fig. 4). When the site of cut was illuminated, a response of positive type was recorded, and when the light spot illuminated a point on the other side of the cut at a distance of $0.5 \mathrm{~mm}$. from the electrode, a small potential of similar shape was obtained, but none could be recorded when a point $1 \mathrm{~mm}$. away from the electrode was illuminated.

It is possible that responses recorded outside the illuminated spot are due to stray light, but the experiment of cut in series B of Fig. 4 will be difficult to interpret in terms of stray light. The point 0 at which the electrode was placed was not damaged by the cut, because a normal negative response was obtained to illumination of this point. However, by the same electrode at 0 a response of positive type was recorded when the damaged part at $0.3 \mathrm{~mm}$. was illuminated. Therefore, the potential can not be a response to stray light at the site of the electrode. It must be due to either electrotonic spread from the damaged part or physiological spread.

Pilz et al. ${ }^{12)}$ found responses of positive type at a damaged part which approached the normal type when a neigbboring intact part was illuminated. This may be due to electrotonic spread. In similar experiments made on the anterior side of the isolated retina responses were reduced in maganitude by a cut, but normal in shape. It was, however, uncertain because of the vitreous body, if the important part of the retina was damaged by the cut.

The effect of stray light was eliminated in the following experiments shown in Fig. 5. A represents a control taken under dark adaptation. The lower tracing represents a response at the illuminated site and the upper one a response at a distance of $1 \mathrm{~mm}$. from the illuminated locus. A background illumination of 40 lux reduced both responses (B). Record $\mathrm{C}$ was obtained when the locus from which the upper tracing was obtained was continuously illuminated by a light spot of the same size and intensity as the stimulus light spot. Under such strong continuous illumination the effect of stray light is obviously negligible so that the response represented by the upper tracing can not be due to stray light. For record $\mathrm{D}$ a background illumination of 40 lux was added to the local constant illumination used in $\mathrm{C}$.

These experiments show that background illumination is effective in reducing the magnitude of responses, while a small local adaptation is almost ineffective. If response recorded at a distance of $1 \mathrm{~mm}$. from the stimulated site were due to electrotonic spread, it could be expected that the upper and lower tracings are identical in form. As a matter of fact, however, a considerable difference can be seen between the latencies of both tracings. 


\section{Lissajous' figure of responses at different loci}

Two microelectrodes were arranged on the surface of the inverted retina at a distance of $1 \mathrm{~mm}$. apart to record responses simultaneously. The upper record in Fig. 6A represents responses to flickering light at the illuminated locus and the lower record those obtained from the unilluminated locus. The stimulus was $100 \times 150 \mu$ in size and its intensity 4,000 lux. The frequency of flicker was $18 \mathrm{cps}$, and light to dark ratio was 1:1.

Fig. 5

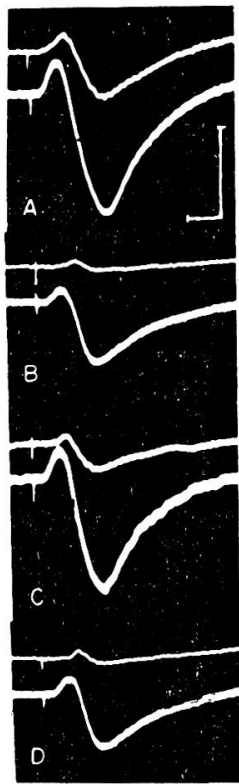

Fig. 6
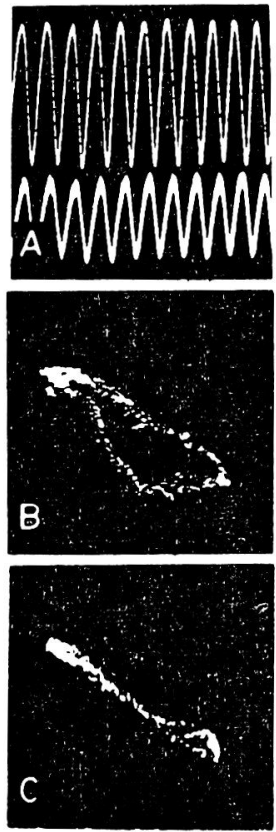

Fig. 5. Simultaneous records from two electrodes arranged $1 \mathrm{~mm}$. apart. Each lower tracing represents response to a light spot $100 \times 150 \mu$ in size at the center of illumination. Stimulus intensity was 4,000 lux. Each upper tracing represents response recorded by other electrode. A : Recorded without background illumination. B : Recorded under background illumination of 40 lux. C : Recorded without background illumination, but the tip of remote electrode was constantly illuminated with light spot of the same size and intensity as stimulus spot. D : Recorded under background illumination of 40 lux, otherwise under same experimental conditions as $\mathbf{C}$. The beginnings of stimuli are indicated by shock-artifact. Vertical bar indicates $500 \mu \mathrm{V}$ and horizontal bar $100 \mathrm{msec}$.

Fig. 6. A : Upper recod indicates response at illuminated locus to intermittent light of $18 \mathrm{cps}$, and lower one response at a distance of $1 \mathrm{~mm}$. B : Lissajous' figure recorded by upper and lower responses in A. C : Lissajous' figure recorded by two responses at points $1 \mathrm{~mm}$. apart subjected to common illumination. 
A Lissajous' figure was recorded with the two kinds of response mentioned above (see B in Fig. 6). The figure indicates that there was a certain phase difference between the response at the illuminated locus and that obtained from the unilluminated neighboring part. For control a Lissajous' figure was recorded by responses to intermittent illumination common to both electrodes. This finding is in line with the experiment shown in Fig. 5.

\section{Dependence of slow potentials on depths}

The potential of positive type can be seen after the superficial layer of the inverted retina has been damaged. If it is assumed that the receptor layer is damaged by this treatment, the potential of this type must originate in some layer other than the receptor layer.
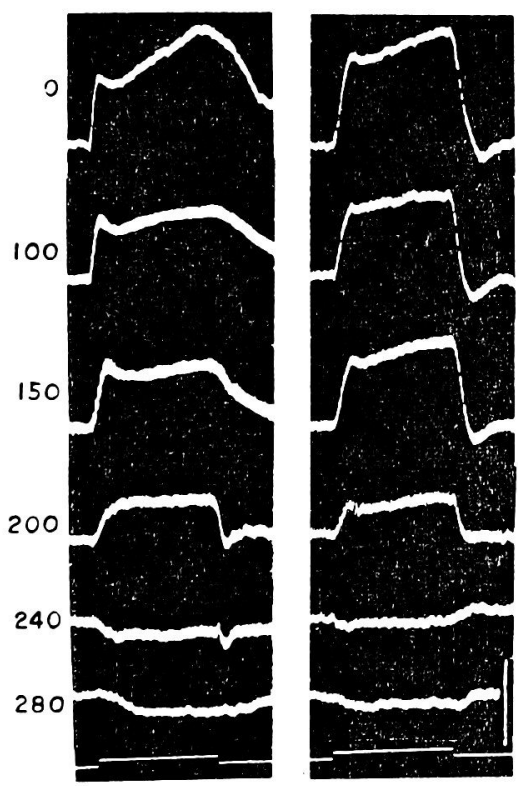

Fig. 7. Responses of positive type at various depths of retina. Left column concerns responses to even illumination and right one responses to microillumination. Numerals indicate apparent depths in $\mu$ from receptor side.

Tomita et al. ${ }^{7)}$ inserted a microelectrode into the retina and found that PIII component which was isolated by potassium reversed its polarity when the electrode reached a depth of a layer external to the bipolar cell layer. He regarded this finding as evidence that the major part of PIII was originated in the bipolar cell layer.

The records in Fig. 7 which were obtained at room temperature of $20^{\circ} \mathrm{C}$ show responses recorded by a microelectrode inserted into the retina 
from the surface of the receptor layer where damage was made with filter paper. The left column represents responses to even illumination and the right one those to microillumination and numerals show depths in $\mu$ from the surface. The "off" swing was slower in records taken from layers near the surface, but such was not a constantly observable finding. When the electrode was inserted to a retinal layer, possibly the inner plexiform layer, at a depth of 200-240 $\mu$ the response was found to reverse its polarity. The location of change in polarity did not show a difference greater than $\pm 30 \mu$ in a number of such experiments. The reverse in polarity suggests that the origin of the response of positive type may be in the inner nuclear layer.

Fig. 8 shows normal responses recorded in similar way from a not

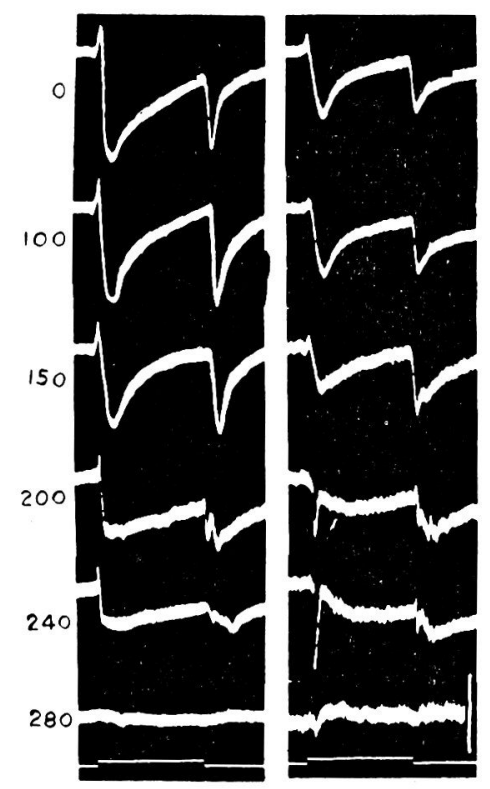

Fig. 8. Responses of negative type at various depths of retina. Left and right columns concern responses to even and focal illuminations respectively.

damaged retina. The response type was negative both to even illumination and microillumination upto the depth of $150 \mu$, but a change occurred at depths of $200-240 \mu$ especially in responses to microillumination, the slow negative response to microillumination was preceded by a spike-like nagative deflection, while such a negative sharp deflection was absent in the response to even illumination. Judging from the latency this negative sharp deflection did not seem to be a reversed on-positive response, and never appeared in the response of positive type. When the light spot was moved $0.5 \mathrm{~mm}$. away from the electrode this deflection was lost sight 
of so that the response became similar to that to even illumination.

When the electrode was advanced into a retina showing responses of positive type to microillumination (see A in Fig. 9) a change in polarity occurred at a depth of about $250 \mu$ from the surface of the receptor side and spikes appeared superimposed on the slow negative potential. (see in Fig. 9).

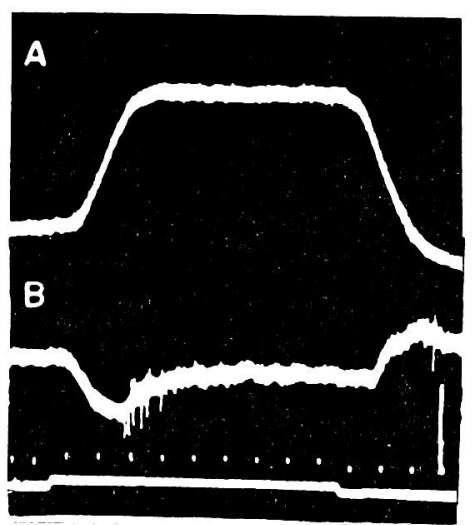

Fig. 9. A : Response of positive type on receptor surface. B : Response accompanied by spikes at a depth of $250 \mu$ from receptor side.

\section{Discussion}

It has been shown above that the responses obtained by a microelectrode to microillumination are focal in character and are different from those of the usual ERG. But local responses to microillumination are always very similar in shape to the ERG when they are recorded from the surface of the inverted retina. So on the receptor surface focal response and ERG may be picked up from the common origin. The additivity in area-effect on ERG found by Brindley ${ }^{13)}$ is one of evidences for this conjecture. But the responses recorded in the depth of more than $100 \mu$ are different from those recorded from the receptor surface. They are intraretinal focal responses.

It is possible that the response of positive type is identical with PIII. When $1 \%$ solution of $\mathrm{KCl}$ is dropped on the inverted retina or when the preparation is laid in an atmosphere containing $10 \% \mathrm{CO}_{2}$ a change in response type occurs from normal negative type to positive one. This change is the same as in isolation of PIII from the ERG. The origin of the response of this type seems to be in the intermediate layers such as the inner plexiform layer and inner nuclear layer. Tomita ${ }^{7)}$ assumed that its origin is in the bipolar cell layer. A response of positive type was obtained even when a shallow cut was made between the stimulus spot and the recording 
electrode on the receptor surface. This finding suggests that a deeper layer must be responsible for PIII.

The phase difference indicated in the Lissajous' figure suggests a possibility of propagation, although farther evidence is needed for this notion.

It has been said that the b-wave and PIII are due to rods and cones respectively. Deane et $a{ }^{10}{ }^{10}$ reported, however, that the cone retina of the turtle had a distinct b-wave and that it was larger in amplitude to red stimulus light than to green one. From their experiment on flicker Dodt \& Enroth ${ }^{14)}$ considered that the a-wave expressed rod activity while the b-potential following high flicker frequency was due to cone activity. In the experiment mentioned above the negative type which was assumed to be identical with the b-wave was obtained more easily in light adaptation than in dark adaptation. This fact seems to suggest that the negative and positive types are associated with cone and rod activity respectively.

It was shown above that even when the response recorded from the receptor surface lacked the negative component, spikes appeared in the record obtained from a deep layer, possibly the ganglion cell layer. So the spikes in this layer seem to be more closely related to the slow potentials of positive type obtainable from the receptor side than to those of negative type. Tomita et $a .^{15)}$ and Gouras ${ }^{2)}$ reported that ganglion cell activity was associated with surface negative waves. They used opened excised eyes, so that the surface-negative wave may be equivalent to the response of positive type on the inverted retina.

It was assumed by Granit ${ }^{11}$ that the off-response of ERG was produced by the return of PIII to the baseline. The off-response could be seen in the response of positive type. If this type of potential is identical with PIII, it is apparent that the cause assumed by Granit is not the only one available for interpretation of the off-response. On the other hand, Tomita ${ }^{7}$ said that one part of the off-response had its origin in a layer common to the b-wave. In the present experiment sometimes a part of the inverted retina showed responses of positive type while the other parts showed those of negative type as mentioned above. In such preparations the off-responses obtained from these parts different in character showed little regional difference.

\section{SUMMARY}

A KCl-filled microelectrode was inserted into toad's inverted retina to record slow potentials to microillumination or to even illumination.

1. Responses obtained from an inverted retina was compared with those recorded from an opened eyebulb, and from this experiment it was suggested that the pigment epithelium contributes to the c-wave of ERG.

2. Responses were divided into two types; positive and negative. 
It was possible to obtain the positive type by giving a slight damage to the receptor surface. The negative type was obtainable more easily in light adaptation than dark adaptation.

3. Properties of focal responses to microillumination were examined. In experiments in which a mechanical cut or twin stimulus spots were used it was confirmed that the spread of potential over $1 \mathrm{~mm}$. from the illuminated focus was not due to stray light. The latency of the response obtained at the illuminated spot was found shorter than that of the response obtained from a point of the surrounding area, as evidenced by comparison of the two records or by Lissajous' figure.

4. Slow potentials of positive and negative types were recorded from different layers of the retina and compared with similar experiments by previous workers.

5. It was discussed on the origin of each component of ERG.

I am greatly indebted to Prof. K. Motokawa for his kind guidance and for many valuable discussions.

\section{References}

1) Granit, R., Receptors and sensory perception. Yale Univ. Press, 1955.

2) Tomita, T., Jap. J. Physiol., 1956, 6, 118.

3) Svaetichin, G., Acta Physiol. Scand., 1953, 29, Suppl., 106, 538.

4) Brindley, G. S., J. Physiol., 1956, 134, 360.

5) Noell, W. K., USAF School of Aviation Medicine, Raldolph Field, Texas, 1953, 122 pp.

6) Svaetichin, G., Acta physiol. Scand., 1956, 39, Suppl., 134, 55.

7) Tomita, T., Funaishi, A. \& Shino, H., Jap. J. Physiol., 1951, 2, 147.

8) Tomita, T., Mizuno, H. \& Ida, T., ibid., 1951, 2, 171.

9) Bernhard, C. G., Acta physiol. Scand., 1942, 3, 132.

10) Deane, H. W., Enroth-Cugell, C., Gougaware, M. S., Neyland, M. \& Forbes, A., JNeurophysiol., 1958, 21, 45.

11) Ottoson, D., Svaetichin, G., Cold Spr. Harb. Symp. Quant. Biol., 1952, 17, 165.

12) Pilz, A., Sickel, W., \& Birke, R. Pflüg. Arch. Physiol., 1958, 265, 550.

13) Brindley, G. W., J. Physiol., 1958, 140, 247.

14) Dodt, E., Enroth, C., Acta physiol. Scand., 1954, 30, 375.

15) Gouras, P., Amer. J. Ophthalmology, 1958, 46, 59. 